\title{
Defects in III-Nitride Microdisk Cavities
}

\author{
C. X. Ren ${ }^{1}$ *, T. J. Puchtler ${ }^{1 \uparrow}$, T. Zhu ${ }^{1}$, J.T. Griffiths ${ }^{1}$, R.A. Oliver ${ }^{1}$ \\ 1. Department of Materials Science and Metallurgy, Charles Babbage Road, Cambridge, CB3 0FS, United \\ Kingdom \\ ${ }^{\uparrow}$. Current address: Department of Physics, University of Oxford, Oxford, OX1 3PU, United Kingdom
}

\section{Abstract}

Nitride microcavities offer an exceptional platform for the investigation of light-matter interactions as well as the development of devices such as high efficiency light emitting diodes (LEDs) and lowthreshold nanolasers. Microdisk geometries in particular are attractive for low-threshold lasing applications due to their ability to support high finesse whispering gallery modes (WGMs) and small modal volumes. In this article we review the effect of defects on the properties of nitride microdisk cavities fabricated using photoelectrochemical (PEC) etching of an InGaN sacrificial superlattice (SSL). Threading dislocations originating from either the original GaN pseudosubstrate are shown to hinder the undercutting of microdisk cavities during the photoelectric chemical (PEC) etching process resulting in whiskers of unetched material on the underside of microdisks. The unetched whiskers provide a pathway for light to escape, reducing microdisk Q-factor if located in the region occupied by the WGMs. Additionally, dislocations can affect the spectral stability of quantum dot emitters, thus hindering their effective integration in microdisk cavities. Though dislocations are clearly undesirable, the limiting factor on nitride microdisk Q-factor is expected to be internal absorption, indicating that the further optimisation of nitride microdisk cavities must incorporate both the elimination of dislocations and careful tailoring of the active region emission wavelength and background doping levels. 


\section{Introduction}

III-nitride materials have been at the centre of a revolution in optoelectronics in the past two decades. Although this family of materials is perhaps most renowned for their central role in the development of high-brightness white light emitting diodes (LEDs) [1], they are also promising materials for various other optoelectronic devices. GaN-based microcavities can facilitate the development of exciting new devices such as resonant cavity LEDs [2], vertical cavity surface-emitting lasers (VCSELs) [3], single photon sources (SPSs) [4], room-temperature polariton lasers [5,6], micro- and nanolasers [7-9] and platforms for studies in cavity quantum electrodynamics [10].

Despite many successful commercial and technological applications in optoelectronics, III-nitride devices must contend with extremely densities of extended defects, particularly dislcations, attributed to the large lattice mismatch between GaN and commonly used substrates such as sapphire or silicon [11]. As such, the study of effects of defects on III-nitride devices is crucial to their improvement and optimisation. This review will focus on the effects of defects on III-nitride microdisk cavities, an application of III-nitride materials which has garnered considerable attention in the past years $[7,12-$ $16]$.

\subsection{Microcavities}

To provide context for out later discussions, we first introduce the concept of microcavities in general and discuss their properties and potential applications.

Microcavity emitters possess rather singular optical properties due to their dimensions. By matching one or more dimensions of the cavity to the order of the wavelength of confined light a plethora of effects can be produced such as low-threshold lasing, directional luminescence and enhanced nonlinear conversion [5]. By confining a dipole within a microcavity, one can modify its emissive properties by altering the photon density of states. The interaction rate between the confined dipole and a cavity photon relative to the average rate of dissipation of a cavity determines whether the microcavity operates in the weak-coupling or strong-coupling regime 
Weak coupling occurs when dissipation overwhelms the dipole-cavity photon interaction: in essence, the effect of the microcavity in this case is to alter the vacuum description of the dipole lifetime, resulting in an increase in spontaneous emission for on-resonance cavity modes, known as the Purcell effect [17]. Weakly-coupled microcavity systems have applications across a wide range of optoelectronic devices due to this effect: from enhancing the recombination rate and extraction efficiency of embedded single photon emitters [18] to the development of high efficiency, low threshold lasers [14].

An interaction occurring on shorter timescales than the average dissipation rate of the cavity photon is defined as being in the strong coupling regime, and results in the formation of admixed eigenstates populated by quasiparticles known as polaritons, which are hybrid particles combining a photon and an electric dipole. The bosonic nature of these quasiparticles has led to the observation of spontaneously emitted coherent light from condensates of exciton-polaritons, a phenomenon also known as polariton lasing [19]. The expected threshold energy for coherent emission from a polariton laser is expected to be much smaller than that of a conventional laser due to the lack of the requirement of population inversion, thus rendering polariton lasers extremely attractive as lowthreshold lasing applications [5]. Beyond polariton lasing, strong coupling in microcavities is also required for key quantum information processing tasks such as the entanglement of distinguishable quantum systems and controlled coherent coupling[20,21].

A crucial characteristic of an optical cavity is known as its quality factor, or Q-factor. This is simply a measure of the rate of decay of a resonant mode within the cavity through radiative, absorption and scattering losses, where $\frac{1}{Q}$ is the proportion of energy lost during a single round-trip of the cavity. By examining the emission spectrum of a cavity, one can extract the respective Q-factors of the modes using the equation:

$$
Q=\frac{\lambda_{\text {cav }}}{\Delta \lambda_{\text {cav }}}
$$


Where $\lambda_{\text {cav }}$ is the cavity mode wavelength and $\Delta \lambda_{\text {cav }}$ is the full width at half maximum (FWHM) of the mode. Reported values in literature are typically the highest extracted Q-factor for all observed modes. Whilst there are a number of metrics for the performance of microdisk cavities, we will here focus on Q-factor since it can be related quite directly to the loss mechanisms which lead to decay of the relevant mode.

\subsection{III-nitride Microcavities: Opportunities and Challenges}

III-nitride microcavities with embedded optical emitters are highly sought after due to the favourable properties of nitride materials such as low surface recombination velocities [22], high exciton binding energies (from $20 \mathrm{meV}$ for bulk $\mathrm{GaN}$ [23] to $36 \mathrm{meV}$ for GaN quantum dots (QDs) [24]) and direct bandgaps spanning the ultra-violet and visible wavelengths and into the infra-red [25-27], tailored by alloying. These favourable properties have enabled progress with InGaN emitters in many areas such as low threshold lasing [14], observations of Rabi oscillations [28] and cavity enhanced single photon emission [18], all in the blue-green spectral range. Although polariton lasing has been demonstrated in several material systems such as InGaAs/GaAs quantum wells (QWs) [29,30] and CdTe QWs [31,32] the thermal broadening of the exciton resonances beyond cryogenic temperatures in these materials exceeds their respective binding energies, preventing the realization of practical polariton laser devices capable of operating at room temperatures. III-nitride based microcavities have enabled the demonstration of room-temperature polariton lasing in both optically [5] and electrically injected [6] configurations due to the large oscillator strength and exciton binding energies inherent to this material system [19].

Despite such promising properties and progress, inherent chemical, structural and electrical properties of III-nitride materials present severe challenges in terms of the realisation of optical devices perhaps best evidenced by the fact quality factors for III-nitride cavities are typically at least an order of magnitude lower than in other III-V systems [33-35]. Challenges with cavity fabrication include the 
very high chemical and thermal stability of $\mathrm{GaN}$ and InGaN-based materials, leading to difficulties in both dry and wet-etching [36]. The deviation of the GaN unit cell from an ideal tetrahedral structure also results in inherent polarization fields within III-nitrides which not only affect the emissive properties of embedded emitters in cavities but are also sensitive to carrier density and strain [37], hindering efficient cavity coupling [38,39]. Issues arising from chemical and electrical properties of the material system are further compounded by the inherently high dislocation densities in heteroepitaxially grown GaN due to lattice mismatched substrates [40]. Sapphire, which is the most commonly used substrate, has a large mismatch of $\sim 17 \%$ with $c$-plane GaN resulting in typical threading dislocation densities as in the range of $10^{8}-10^{10} \mathrm{~cm}^{-2}$ [41]. It is important to note that the origin of threading dislocations is still debated, though their presence is strongly linked to lattice mismatch [11]. Though dislocations in general are expected to have deleterious effects in terms of the electrical and optical properties of devices [11], their effects are device-dependent. Some devices fabricated from highly defective III-nitride materials, for example LEDs, demonstrate performance which is remarkably robust to the presence of dislocations [42], [43,44] whilst others such as IIInitride laser diodes suffer dislocation-induced degradation due to the high electrical and optical powers used in their operation [45].

\subsection{Microdisk Cavities}

Various geometries of nitride-based microcavities have been investigated for applications such as those detailed in section 1.2 including Fabry-Perot resonators [27], [46,47], 2-D and 1-D photonic crystal cavities $[8,9,35,48,49]$ and microdisks $[7,10,50]$. This paper will focus on the microdisk geometry for nitride cavities.Such microcavities are of particular interest, since they can be fabricated using relatively straightforward photolithographic techniques but nonetheless have a small modal volume and are able to support high quality whispering gallery modes [51]. Effective cavities require minimal optical loss, as this reduces the strength of internal optical fields, leading to the requirement of high reflectivities at cavity boundaries. Microdisk, ring and sphere cavities achieve light confinement through total internal reflection, leading to the propagation of whispering gallery modes 
around the periphery of the disk, making microdisks a highly desirable platform through which to study low-threshold lasing and cavity QED in the nitrides.

The figure below illustrates the first order whispering gallery mode for a $1.2 \mu \mathrm{m}$ microdisk calculated by finite difference time domain (FDTD) simulations. Note how the regions corresponding to areas of high field density (red) are confined to the periphery of the microdisk which is outlined in black.

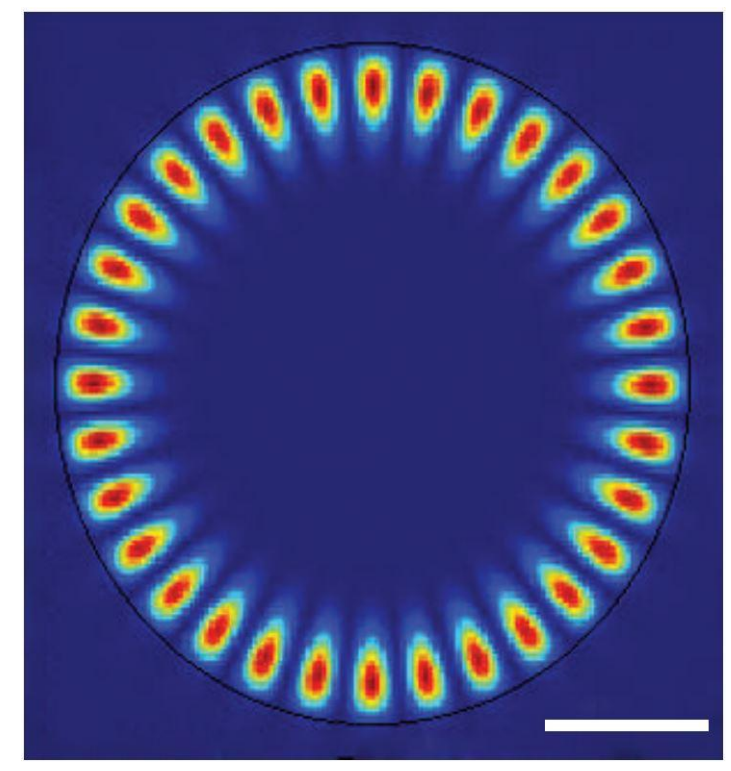

Figure 1: FDTD simulation showing a first order WGM for a $1.2 \mathrm{~m}$ microdisk. Red corresponds to areas of high field density, and blue to the lowest. The edge of the microdisk is delineated in black. Adapted from [7]

\subsection{Microdisk Fabrication}

Despite the expected advantages offered by microdisks, the fabrication of high quality nitride microdisks has proved to be a rather challenging endeavour due to the lack of a convenient wet etching process capable of forming thin disks optically isolated from higher index materials, unlike for the III-V arsenide system [52]. The methods used for nitride micro disk fabrication fall into two main families. Most common are methods which employ a selective wet etch to undercut the disk, where the etched layer may be an AlInN interlayer[53], an InGaN sacrificial superlattice (SSL) [54] (which must be etched by a photoelectric chemical (PEC) method) or the silicon on which some microdisk epitaxy is grown[55]. Alternatively, although less common, bottom-up growth of GaN microdisks has been performed both by RF-plasma-assisted molecular beam epitaxy (RF-PA-MBE) 
[56] and by MOVPE on zinc oxide grown on graphene [57].. Microdisks formed by these various methods are shown in Fig. 2..

a)

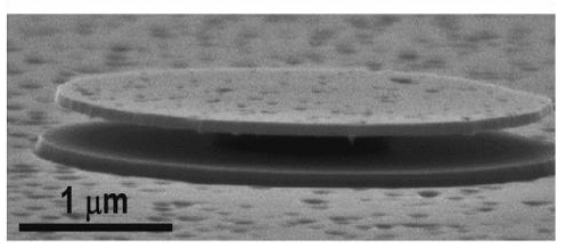

b)

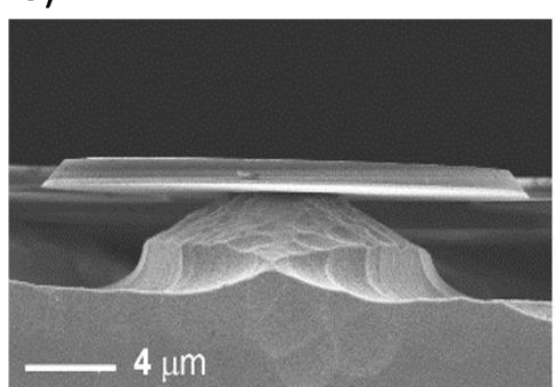

c)
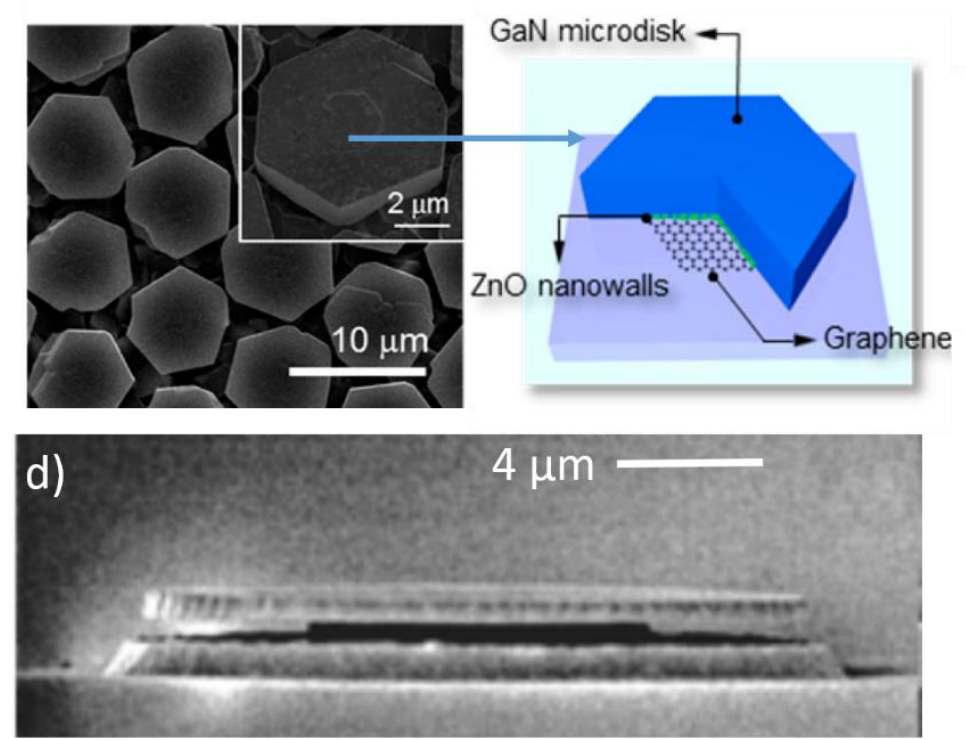

Figure 2: Microdisks fabricated by a) selective etching of a sacrificial AlInN interlayer [58] b) growth on silicon and chemical etching [55] c) growth on ZnO nanowalls grown on graphene [57] and d) PEC etching of an InGaN sacrificial superlattice [59].

In this paper we will address the PEC etching method in particular, as it has resulted in some of the highest reported quality-factors for III-nitride microdisks thus far [10,50], whilst also providing insights into the mechanisms by which defects influence cavity performance [60].

Early efforts in microdisk nitride fabrication involved dry-etching processes, utilising the refractive index mismatch between the light emitting GaN layers and the sapphire substrate to confine light [7]. Although stimulated emission and lasing was observed by Chang et al. in dry-etched GaN microdisk cavities [61], Haberer et al. [59] reduced the required excitation power densities for lasing by an order of magnitude by employing photoelectrochemical (PEC) etching to undercut $\mathrm{GaN}$ microdisks, thus providing superior optical confinement due to the index contrast of the GaN/air interface relative to the GaN/sapphire interface used by Chang et al [61]. Further improvements in fabrication were achieved by Tamboli et al. with room temperature lasing achieved in $\mathrm{GaN} / \mathrm{InGaN}$ microdisks through enhancements in microdisk circularity and sidewall smoothness [7]. 
A typical workflow for microdisk fabrication using PEC etching is shown in Fig.3 below: a sample structure containing InGaN active layers in a $\mathrm{GaN}$ membrane, with an $\mathrm{InGaN}$ sacrificial superlattice is grown on a GaN template. This structure is then masked using $\mathrm{SiO}_{2}$ microbeads, which act as a hard mask for the inductively coupled plasma (ICP) etching which results in circular micropillars. Metallic contacts are then deposited, and the sample is submerged in a weakly acidic electrolyte, such as diluted $\mathrm{HCl}$ or $\mathrm{KOH}$. PEC etching is then used to undercut the sacrificial superlattice whilst preserving the top membrane, resulting in undercut microdisks.

a)

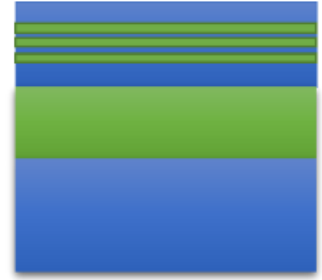

c)

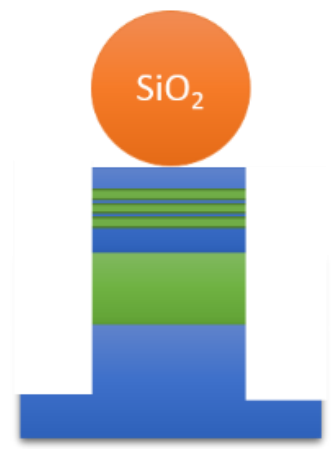

e)

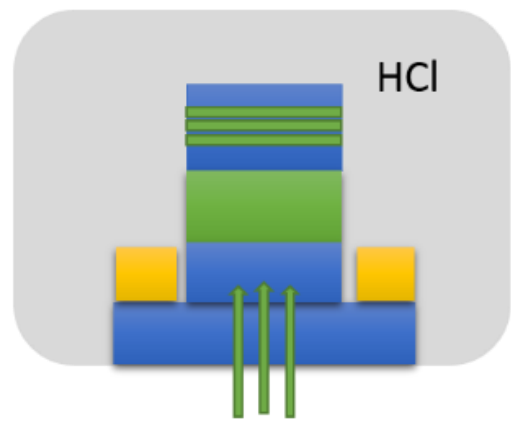

hv b)

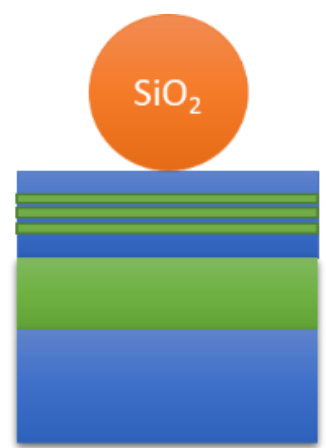

d)

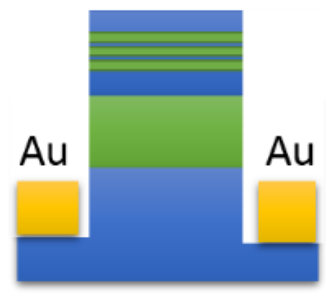

f)

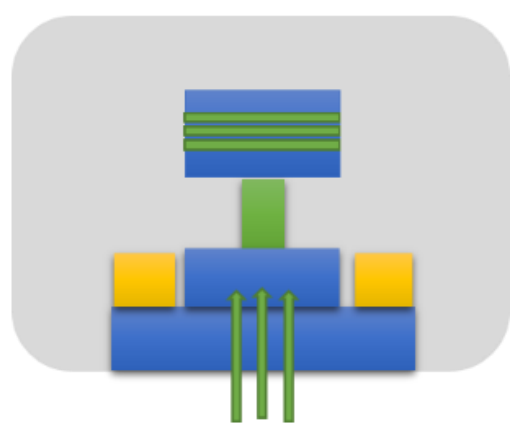

hv

Figure 3: Microdisk fabrication process: a) Sample structure grown b) Masked using silica beads c) ICP etched d) Metallic contacts deposited for PEC etching e) Illuminated for PEC etching using sub-GaN-bandgap energy light f) InGaN SSL 


\subsection{Photoelectric Chemical Etching}

This section will discuss PEC etching in detail as it is the fabrication method used in most studies covered by this review, as discussed in section 1.4.

A typical PEC etching set-up is shown in the Fig. 4:

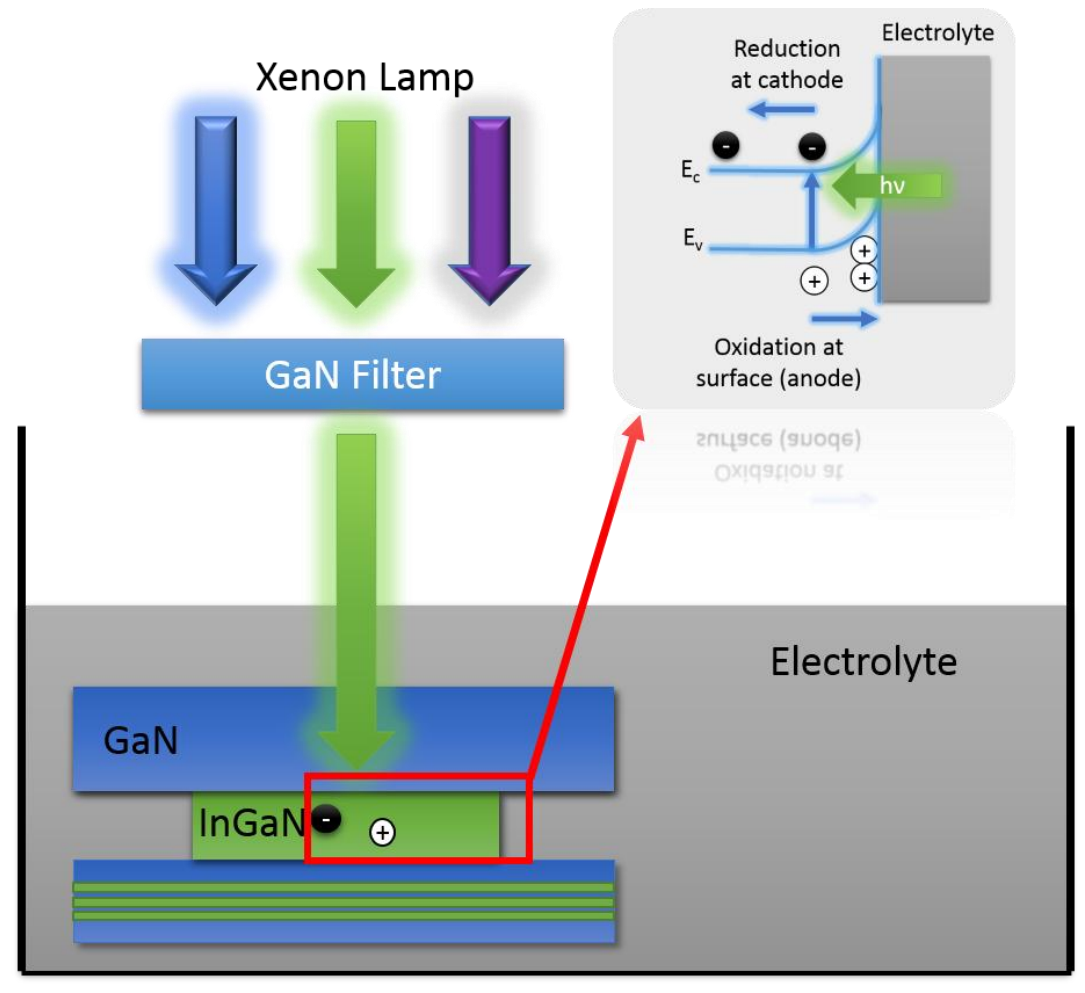

Figure 4: PEC etching set up and charge transfer processes.

PEC etching was first described for III-nitrides by Minsky et al.[62], and is an extremely effective method to perform selective etching as it can be defect-selective $[60,63,64]$, dopant-selective [65] and bandgap-selective $[63,66]$. In the case of III-nitride microdisk undercutting, a GaN filter is used to absorb light emitted by a Xenon lamp, allowing light of wavelength longer than $~ 360 \mathrm{~nm}$ to interact with the structure thus ensuring the majority of carriers are generated in the sacrificial InGaN layer. Holes generated by the incident light diffuse toward the surface of the InGaN layer which in this case acts as an anode whilst the electrons diffuse toward to the metal cathode due to both the overall potential in the connected cell and the band-bending occurring at the surfaces. 
The excess concentration of holes at the sacrificial layer surface drives the oxidation of InGaN, forming $\mathrm{Ga}_{2} \mathrm{O}_{3}, \mathrm{In}_{2} \mathrm{O}_{3}$ and $\mathrm{N}_{2}$, while at the cathode a reduction process is driven by the excess electrons. The oxide generated by the reduction process is then dissolved in the electrolyte, thus etching the sacrificial layer. It is clear that the etch rate of the sacrificial layer is governed by several factors: the rate of generation of the photo-generated electron hole pairs, their diffusion rates towards the anode or cathode, and the concentration of the electrolyte ( $\mathrm{HCl}$ in this case). It is due to this photogenerated carrier concentration dependence that the PEC etching process is defect and dopant selective [60].

\subsection{Whisker Generation in Photoelectric Chemical Etching}

PEC etching can be bandgap selective, a property exploited in the fabrication of a variety of photonic devices which require selective undercutting $[9,10]$, but is also heavily affected by the presence of dislocations [60].

Youtsey et al. demonstrated that dislocations impede the progress of PEC etching and used this effect as a method of determining the dislocation density of $n$-type GaN films: unetched material (or 'whiskers') remained at dislocation sites post-etching due to the electrically active nature of dislocations in GaN, as shown in Fig. 5. It was confirmed that both mixed and edge dislocations result in the formation of whiskers through cross-sectional TEM [60]. Lazar et al. also reported that both screw dislocations and nanopipes associated with coreless screw dislocations results in the formation of whiskers in PEC etching [67]. Thus, it can be concluded that all types of dislocations result in the formation of whiskers in PEC etching. 


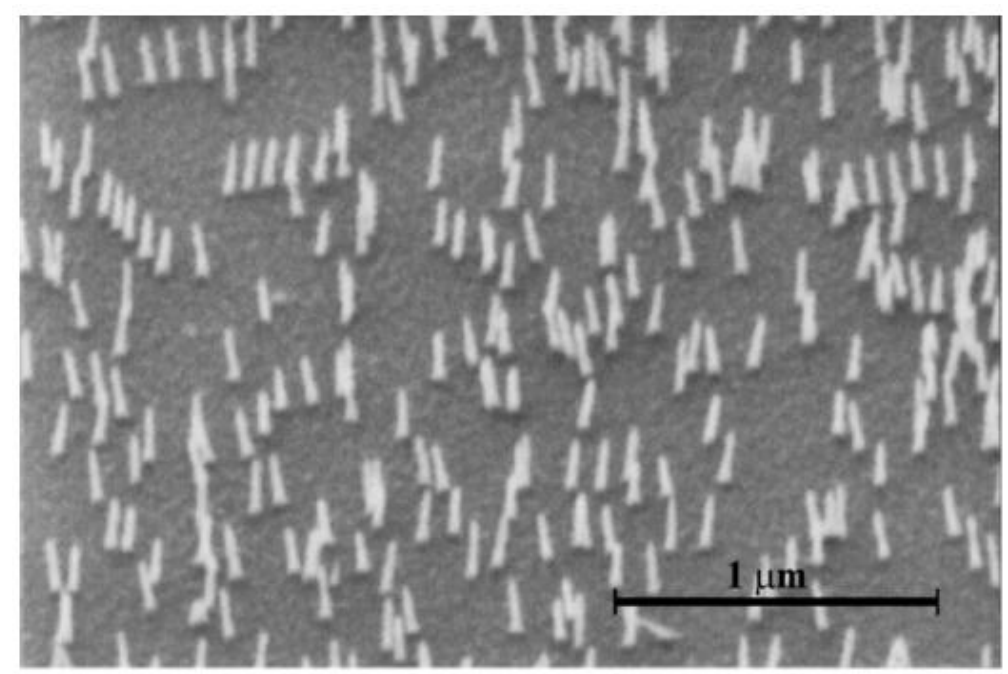

Figure 5: Secondary electron image taken in an SEM of whiskers produced by etching of dislocations in an MOCVD GaN film grown on a silicon carbide (SiC) substrate [60].

The mechanism through which the presence of dislocations impedes the PEC etching process relates to the charge trapping property of TDs: it has been suggested based on cathodoluminescence (CL) studies that TDs act as non-radiative recombination centres $[68,69]$. In this case, photogenerated holes in the vicinity of TDs would be prevented from diffusing to the epilayer surface thus resulting in unetched material. There is also evidence for negatively charged TDs in $n$-type GaN, which can lead to hole-trapping at TDs [70] presenting an alternative or additional explanation for the reduction in surface hole concentration and thus reduced etch rate in areas adjacent to TDs [60].

\subsection{Evidence for Dislocation Induced Whiskers in Microdisks}

In the PEC undercutting of microdisk structures, the lack of etching around dislocations leads to the presence of whiskers protruding from the underside of the disk, as shown in Fig. 6. 


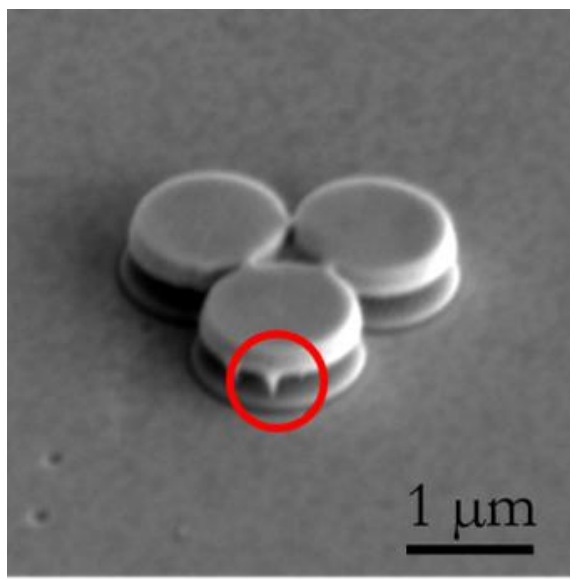

Figure 6: SEM micrograph of a PEC undercut microdisk with a whisker circled in red.

We have developed a dual beam SEM/ focussed ion beam (FIB) based sample preparation method which has allowed for the direct observation of a TD within a whisker from a microdisk using TEM. As the microdisks studied here are extremely sensitive to ion-beam damage and the whiskers are < $100 \mathrm{~nm}$, standard FIB sample preparation procedures are unsuitable for the extraction of single whiskers from already fabricated microdisks.

In order to isolate a whisker on a disk for TEM examination, a target whisker is first identified and the sample rotated in order to ensure optimal positioning of the whisker in the lamella which is lifted-out. A thick $(\sim 2 \mu \mathrm{m})$ protective layer of $\mathrm{Pt}$ is then deposited by electron beam on the surface of the microdisk. It is crucial to avoid using the ion-beam in this step, as the extremely thin microdisk membrane $(<300 \mathrm{~nm})$ can be swiftly damaged and milled away even during the deposition of protective Pt at low currents. A further benefit of using electron-beam deposited Pt is the superior contrast between the protective layer and the microdisk observable in SEM image during thinning. Trenches are milled using the ion-beam on either side of the sample and an Omniprobe ${ }^{\mathrm{TM}}$ is attached to the sample, the sample is then released using the ion-beam and lifted-out using the Omniprobe. The extremities of the lift-out are then thinned gradually using the FIB, whilst the lamella itself is carefully monitored in the SEM in order to stop the thinning process before damaging the whisker. As typical FIB-prepared TEM sample thicknesses are below $100 \mathrm{~nm}$ [71], the initial positioning of the whisker and monitoring during thinning are crucial to the successful preparation of the sample. The steps 
described here were performed on the whisker denoted by the red circle in Fig. 6 and are shown in Fig.

7.
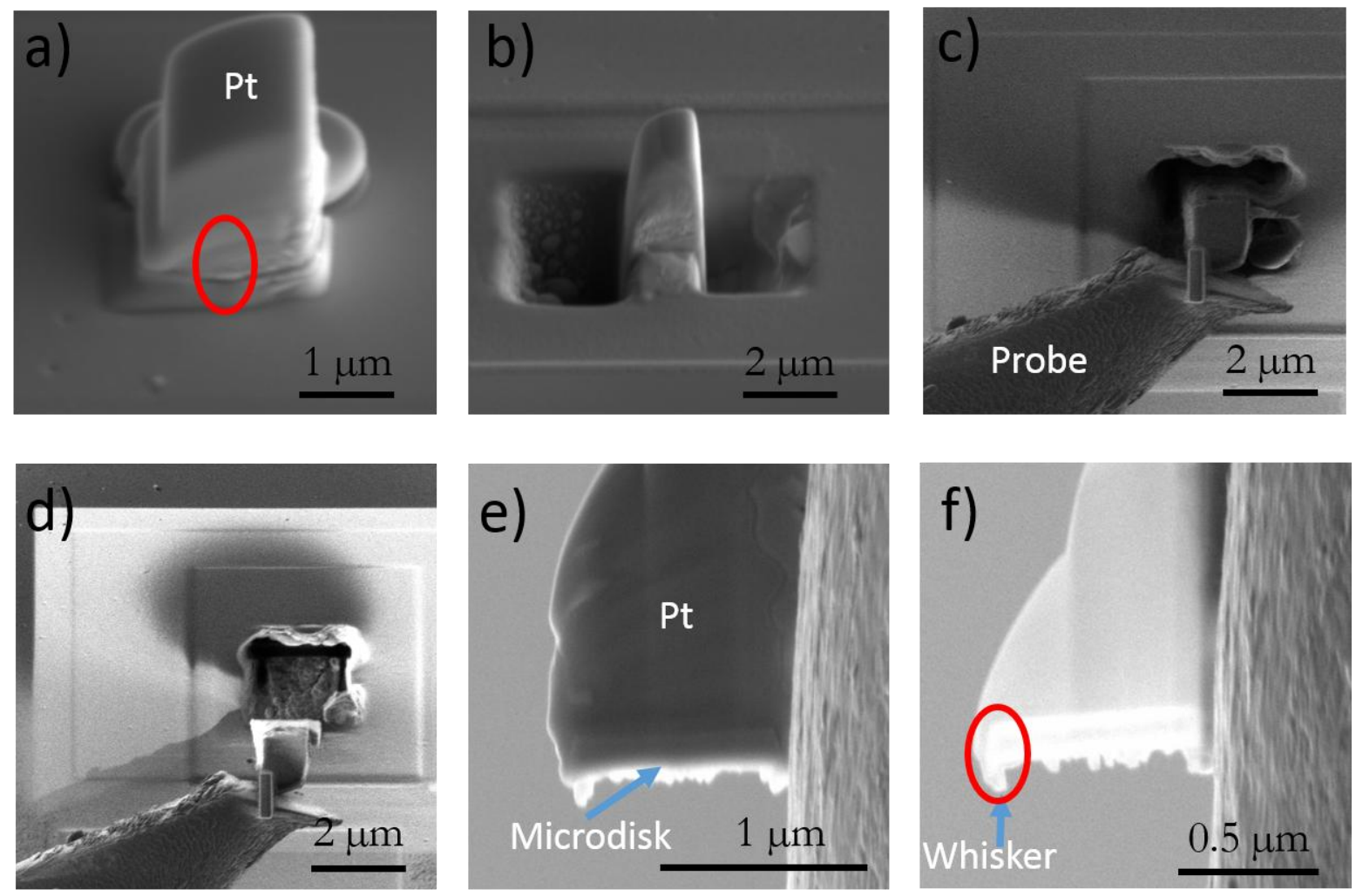

Figure 7: Whisker lift-out process: a) SEM electron beam deposited platinum with the approximate position of the whisker as shown in Fig. 6 circled in red b) milling of trenches by ion beam c)ion-beam image taken after attaching the lift-out probe to the sample using ion-beam deposited Pt and of d) sample release and lift-out e) SEM image of the initial stages of thinning and f) the final stages of thinning with the whisker visible (red ellipse).

Following this, the dislocation was identified and characterised using scanning transmission electron microscopy with a high-angle annular dark-field detector (STEM-HAADF) and WBDF-TEM as shown in Fig. 8 a) and b). A region of the microdisk directly above the whisker has been partially milled away during low energy milling of the sample to reduce the impact of ion beam damage. The dislocation observed here is shown to be a pure edge type dislocation, as demonstrated by the bright contrast produced by the TD observable when activating $\mathbf{g}=\langle 11 \overline{2} 0\rangle$ which is absent when activating $\mathbf{g}=<0002>$ as shown in Fig. 8 a) and 8 b) respectively. Screw and mixed dislocations [64] are also expected to hinder the PEC etching process in a similar fashion and generate whiskers. 
a)

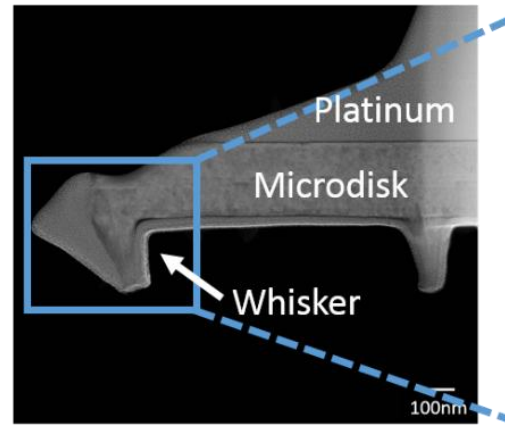

b)

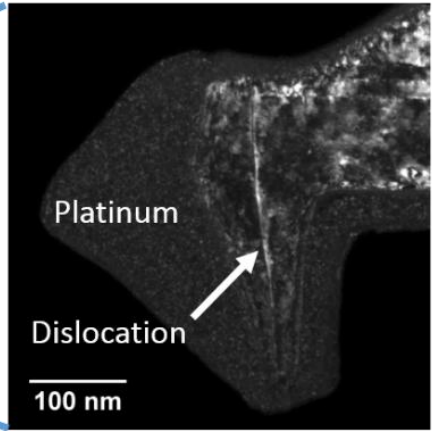

c)

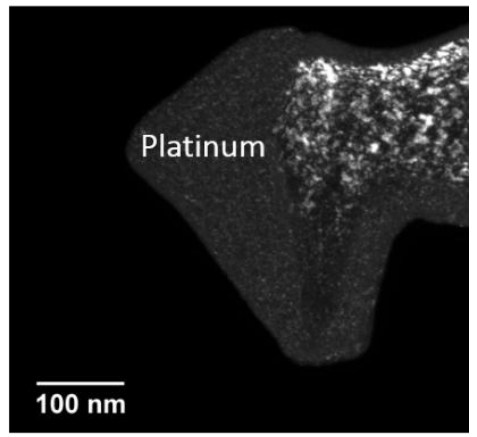

Figure 8: TEM analysis of microdisk whisker by: a) STEM-HAADF, b) WBDF activating $g=<11 \overline{2} 0>\mathrm{f}$ c) WBDF activating $\boldsymbol{g}=\langle 0002\rangle$. The dislocation is only visible along the direction, confirming its nature as a pure edge dislocation. The sample preparation process resulted in part of the microdisk being milled away, as can be seen directly above the whisker.

To our knowledge, this is the first observation of a dislocation within a whisker resulting from incomplete PEC undercutting of microdisks, and direct evidence that the unetched material on the underside of PEC etched microdisks are the result of dislocations hindering the etching process.

We envision this type of dual-beam based technique for the extraction and analysis of features on this scale could be extended with the use of various TEM-based analysis methods such as nanocathodoluminescence (nanoCL) [72] or electron tomography [73] for the investigation of specific nano-scale features in devices beyond microdisk cavities, such as photonic crystal cavities $[9,74]$, providing a promising platform for device analysis and optimisation.

\section{Origin of Dislocations in PEC undercut Microdisk Cavities}

In this section we will consider two origins of dislocations in microdisk cavities fabricated by PEC etching. The first of these is the presence of dislocations which thread through the entire structure from the GaN pseudo-substrate. As GaN is typically grown heteroepitaxially due to the prohibitive price of bulk substrates [40], GaN epilayers contain high densities of dislocations as discussed in section 1.2. These dislocations thread through the entire structure during the growth of subsequent layers. Bennett has reviewed the origins of TDs in the pseudo-substrate in great depth [11] and thus we will not address these issues here. However, a second source of dislocations can be the InGaN sacrificial superlattice, as reported by El-Ella et al. [75], which will be discussed in this section. 
Haberer et al. demonstrated that design of the sacrificial layer necessitates careful consideration for PEC etching to occur successfully. PEC etching under equivalent conditions was compared between a structure containing a 'thick' low In content InGaN layer and an InGaN superlattice consisting of 20 $\mathrm{nm}$ regions of alternating InGaN layers of higher and lower indium content [54]. The thick InGaN layer underwent slow, non-uniform etching due to insufficient carrier confinement which allowed photogenerated carriers to diffuse and recombine before causing etching. The InGaN SSL offered stronger carrier confinement, and also a more uniform distribution of holes across the SSL thickness, due to its modulation of the valence band edge. This allowed for fast, uniform etching during the PEC etching process. This is absolutely crucial in mitigating the polarization fields inherent to $c$-plane nitrides which would otherwise result in etching at only one interface of the sacrificial layer [76]. As a result, the InGaN SSL design proposed by Haberer et al. with thin alternating layers of varying In content has become a commonly used sacrificial structure for the undercutting of microdisks by PEC etching $[7,10,13,15,50,54,59]$. However, the relaxation of pseudomorphic InGaN epilayers on GaN substrates is known to result in the generation of dislocations [77], which in the case of the InGaN SSL growth can result in a second source of dislocations, separate to those associated with the GaN pseudosubstrate.

- El-Ella et al. first demonstrated that the growth of the SSL can result in generation of dislocations by growing a series of structures containing SSLs of varying In content [75]. The general structure of these samples is shown below:

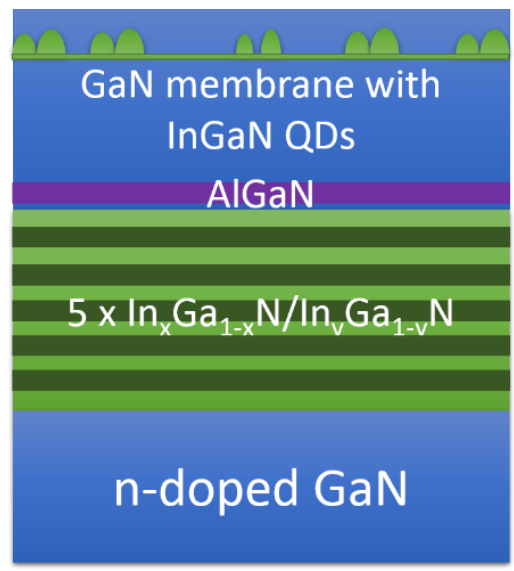


The structures were grown by metal-organic vapour phase epitaxy (MOVPE) and consist of a GaN membrane containing an InGaN QD layer with an $\mathrm{Al}_{0.19} \mathrm{Ga}_{0.81} \mathrm{~N}$ etch stop layer grown on an $\mathrm{In}_{\mathrm{x}} \mathrm{Ga}_{1 \text { - }}$ ${ }_{\mathrm{x}} \mathrm{N} / \mathrm{In}_{\mathrm{y}} \mathrm{Ga}_{1-\mathrm{y}} \mathrm{N}$ SSL on an $n$-doped $\mathrm{GaN}$ pseudo-substrate. For indium contents of $\mathrm{x}=7$ and $\mathrm{y}=10$ in the SSL, transmission electron microscopy (TEM) analysis (see Figure 10) revealed the presence not only of threading dislocations initiating at the pseudo-substrate, but also of additional dislocation loops originating from within the SSL. These dislocation loops included misfit segments in the (0001) plane allowing strain relaxation of the SSL. However, additional experimentation using carefully designed test structures showed that such strain relaxation only occurred in structures with an InGaN quantum dot layer grown above the SSL. An SSL grown without an overlying QD layer did not relax, even when subjected to the same thermal cycles which occurred during QD layer growth. Hence, it was suggested that the SSL itself did not exceed the critical stack thickness for strain relaxation in a multilayer, but the additional strain energy associated with the growth of the QD layer did indeed cause relaxation. The results shown by El-Ella et al. [75] suggested that reducing the strain energy arising in the SSL slightly would prevent dislocation loop formation even with the growth of the QD layer, and indeed, by reducing the indium content in the SSL, it proved possible to achieve a fully strained structure without additional dislocation loops [76]. This illustrates the importance of designing the SSL carefully in the context of the full device structure with which it will be integrated particularly since the extra dislocation loops which were observed in imperfectly designed structures did have a significant impact on cavity performance, as we shall in section 4.1.
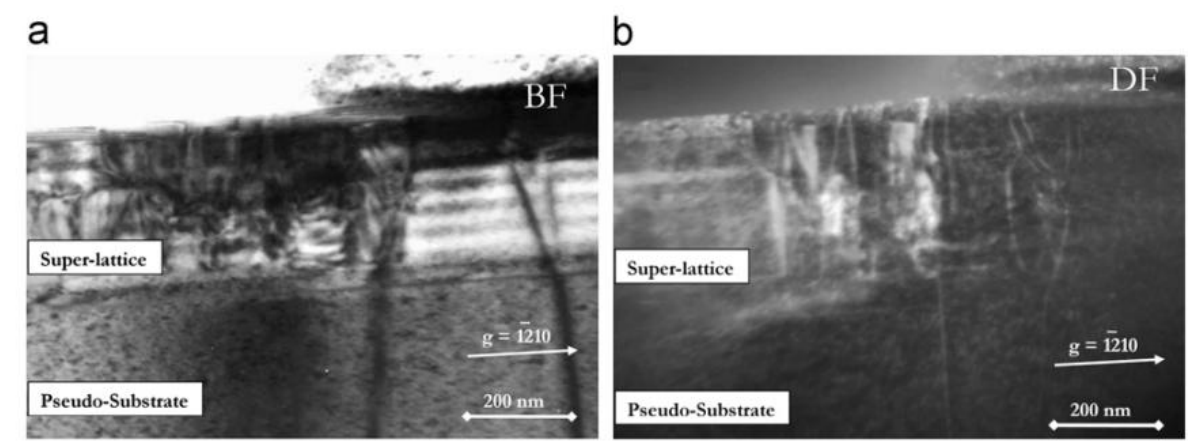

Figure 10: a) Bright-field (BF) and b) weak-beam dark field (WBDF) images of A around $\langle 101 \overline{0}\rangle$ zone axis. Adapated from [75]. 


\subsection{Effect of Whiskers and Dislocations on Microdisk Quality-factor}

As detailed in table 2, by reducing the indium content in the SSL, El-Ella et al. reduced the total density of dislocations penetrating the microdisk membrane by a factor of $>4$.

\section{Structure $\quad$ In (\%) in SSL: $x / y \quad$ Dislocation density $\left(\mathrm{cm}^{-2}\right)$}

\begin{tabular}{l|l|l}
\hline A & $6.7 / 10.7$ & $(3 \pm 0.4) \times 10^{9}$ \\
\hline B & $5.1 / 6.5$ & $(7 \pm 0.7) \times 10^{8}$ \\
\hline
\end{tabular}

Table 1: Summary of In contents (where $x$ and y represent alternating compositions within the same SSL) and pit densities for two structures grown for the fabrication of microdisks by El-Ella et al [76].

Microdisks fabricated from the two samples detailed in the table - sample A with a higher dislocation density and sample B with a lower dislocation density, were examined in room temperature microphotoluminescence (RT- $\mu \mathrm{PL})$. The resulting spectra are shown in Fig. 11, along with SEM images of representative microcavity structures. 

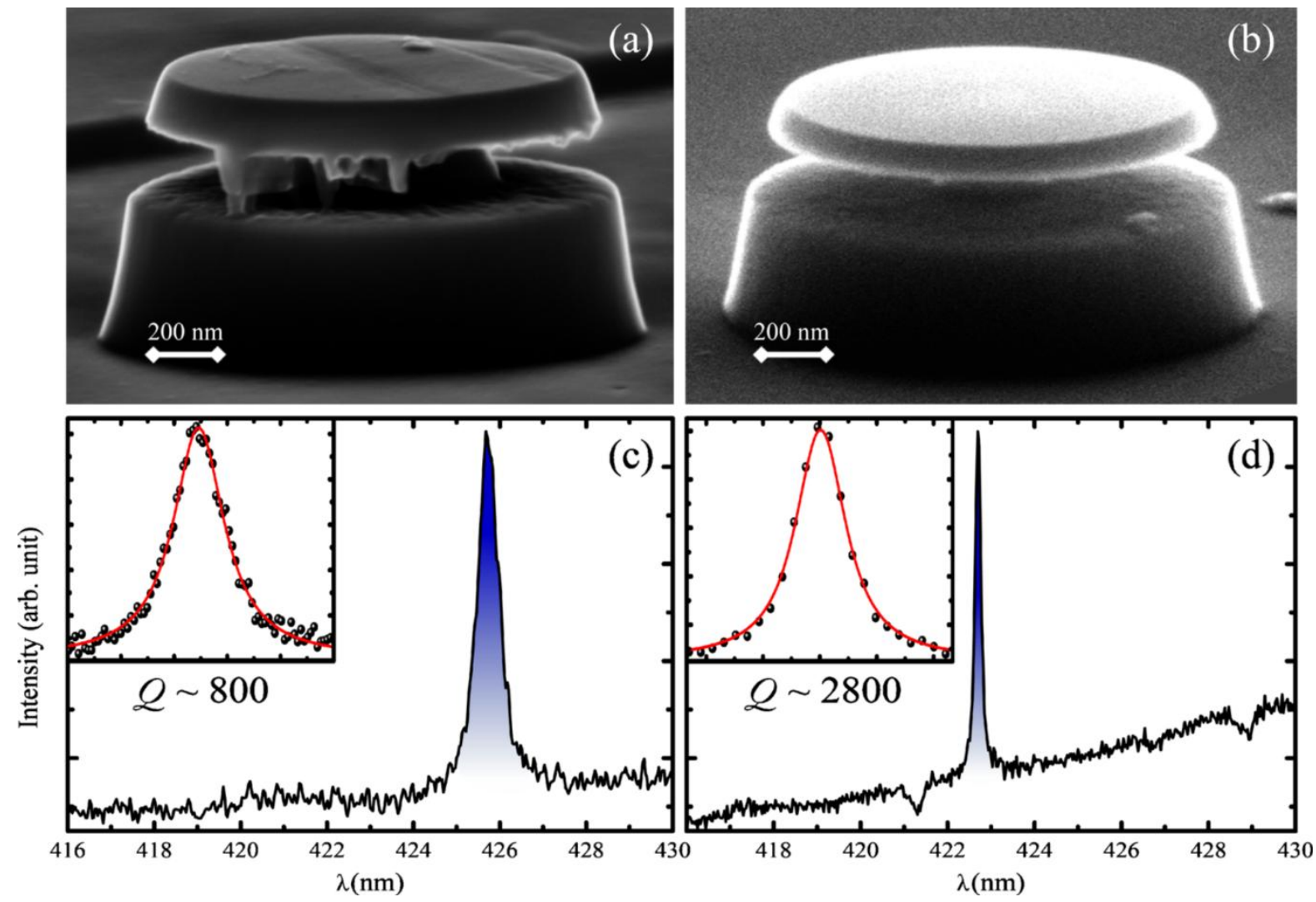

Figure 11: SEM images and RT PL spectra collected from material $\boldsymbol{A}(a, c)$ and $\boldsymbol{B}(b, d)$. Inset graphs show Lorentzian fits of the whispering gallery modes [76].

Microdisks fabricated from sample A exhibited a larger density of whiskers on the underside of the disk. SEM was used to establish that $90 \%$ of disks fabricated from material A exhibited whiskers, compared to only $20 \%$ of disks fabricated from structure B. The difference between the two samples in terms of Q-factor is illustrated in Figure 12 which includes data foreight microdisks fabricated from each sample. 


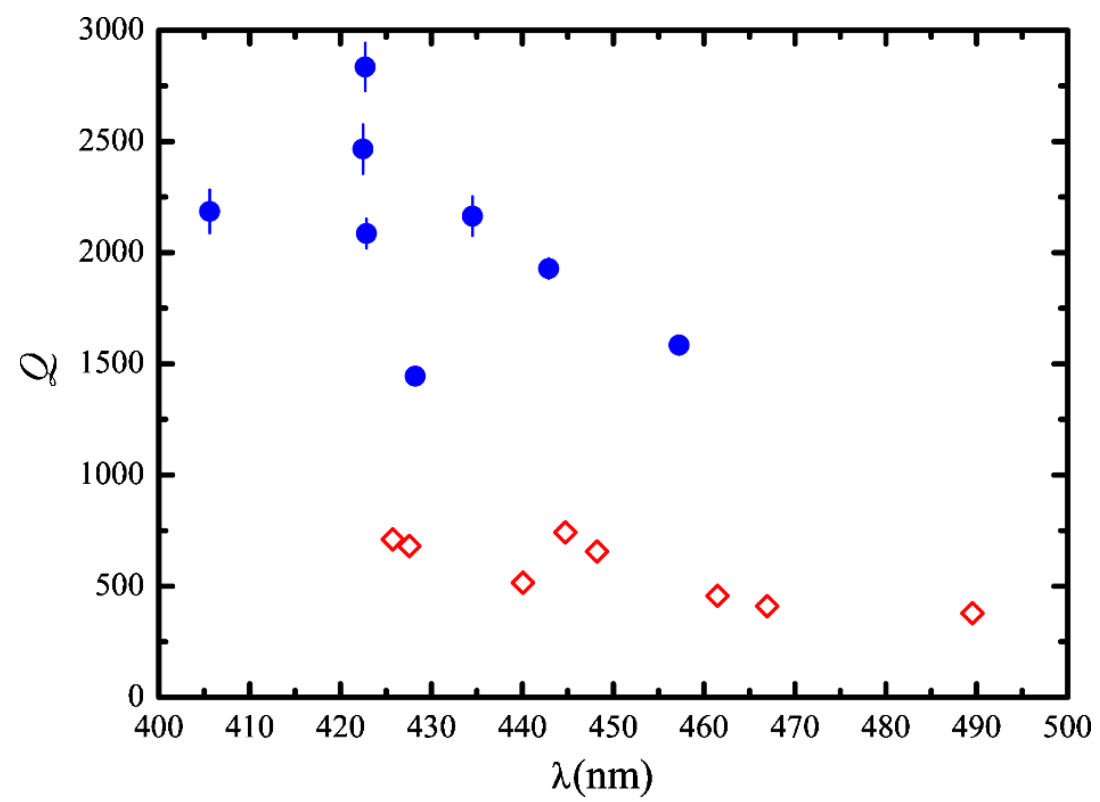

Figure 12: $Q$ values from eight modes of microdisks fabricated from structure $A(\diamond)$ and $B(\bullet)[76]$.

All microdisks fabricated from sample B show a higher Q-factor than all microdisks from sample A, irrespective of variations in wavelemgth. The average Q-factors for microdisks fabricated from samples A and B were 600 and 2000 respectively, suggesting a strong correlation between Q-factor and material quality. Despite these results, the mechanisms behind Q-degradation were not fully investigated [76]. Furthermore, even when grown on strained SSL structures $20 \%$ of the microdisks examined by El-Ella et al. still exhibited whiskers on the underside due to dislocations originating from the GaN pseudo-substrate.

Puchtler et al. addressed these issues by identifying the number and position of TDs in individual microdisk cavities and evaluating the effect of these on the Q-factors of the specific microdisks in question [10]. Unlike earlier studies, this work did not relate average material properties (such as dislocation density) to average device properties, but correlated the performance of each individual device structure with its specific structure. In this study, dark spots in CL images, arising from the reduced radiative recombination in the vicinity of dislocations, were used as a signature for the presence of dislocations, and hence whiskers. The authors first confirmed the correspondence of dark spots in the CL to whiskers by comparing plan-view panchromatic CL images with side-view SEM images of the microdisks. Figure 13 a) shows a typical plan view CL image of a disk, with five dark 
spots labelled. In Figure $13 \mathrm{~b}$ ), the same disk is viewed in SEM from the side, and whiskers corresponding to each of the 5 dislocations are identified. Furthermore, in Figure $13 \mathrm{c}$ ) a direct correlation between the number of dark spots seen in plan-view CL and the number of whiskers seen in side-view SEM is established.
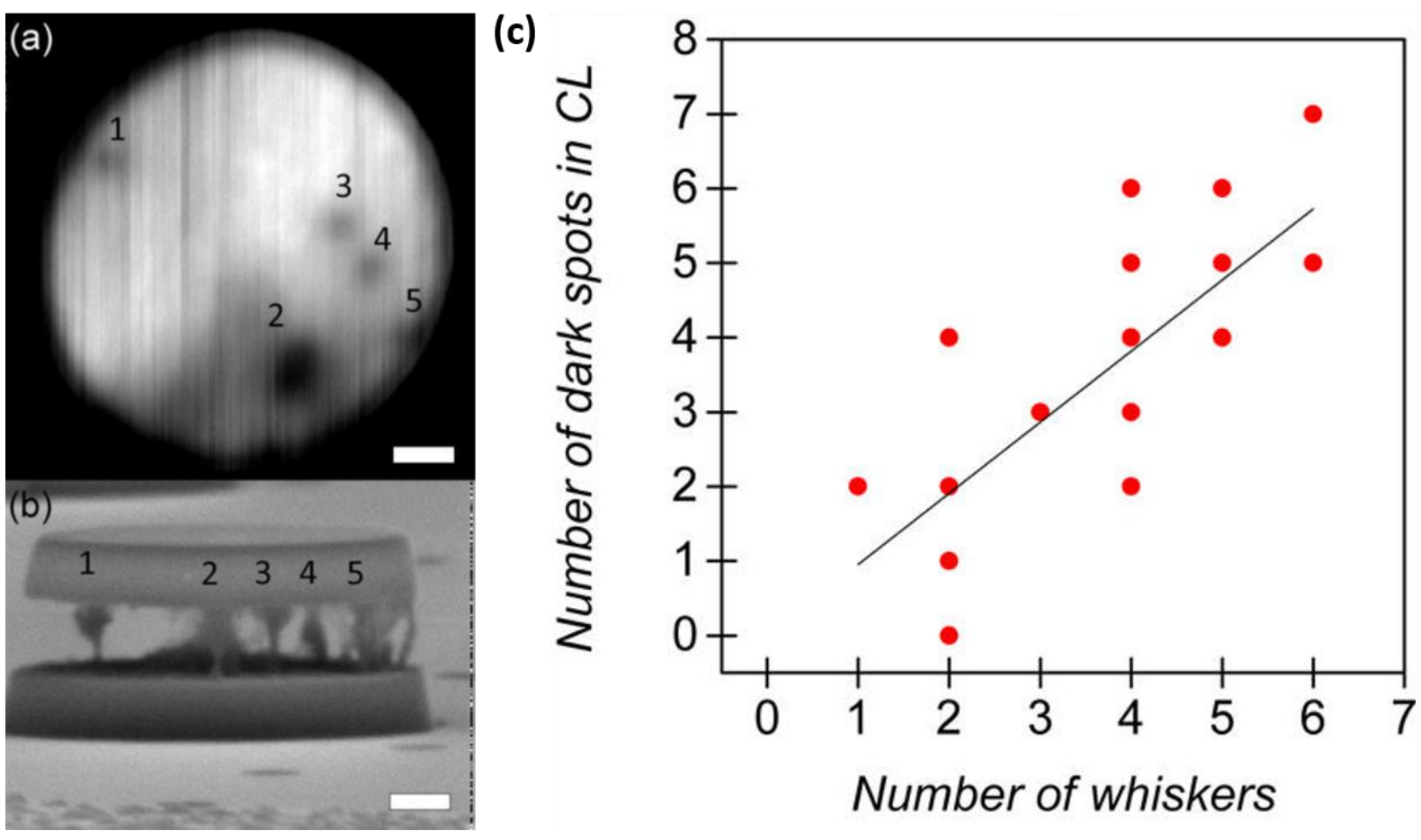

Figure 13:a) Smaple plan-view CL b) Corresponding side-view SEM image of an undercut microdisk with whiskers corresponding to the dark spots shown in a). c) Relationship between whiskers on the underside and dark features counted in plan-view CL. Adapted from [10].

Using plan view CL to measure the number of dislocations per microdisk, the correlation between dislocation number and Q-factor (extracted from the modal peaks in $\mu \mathrm{PL}$ ) was investigated. A reduction in Q-factor with increasing dislocation density was reported (Figure 13(a)), as expected from the results shown in [76]. However when considering TDs within the inner region of the disk (less than $0.4 \mu \mathrm{m}$ from the centre) (Figure $13 \mathrm{~b}$ )) this correlation disappeared. In fact, the relationship between Q-factor and dislocation number is only observed when considering dislocations in the outer periphery of the microdisks (more than $0.4 \mu \mathrm{m}$ from the centre) (Figure $13 \mathrm{c}$ )), suggesting that only dislocations located in the region within which the WGMs are confined play a significant role in limiting microdisk quality factor [10]. 

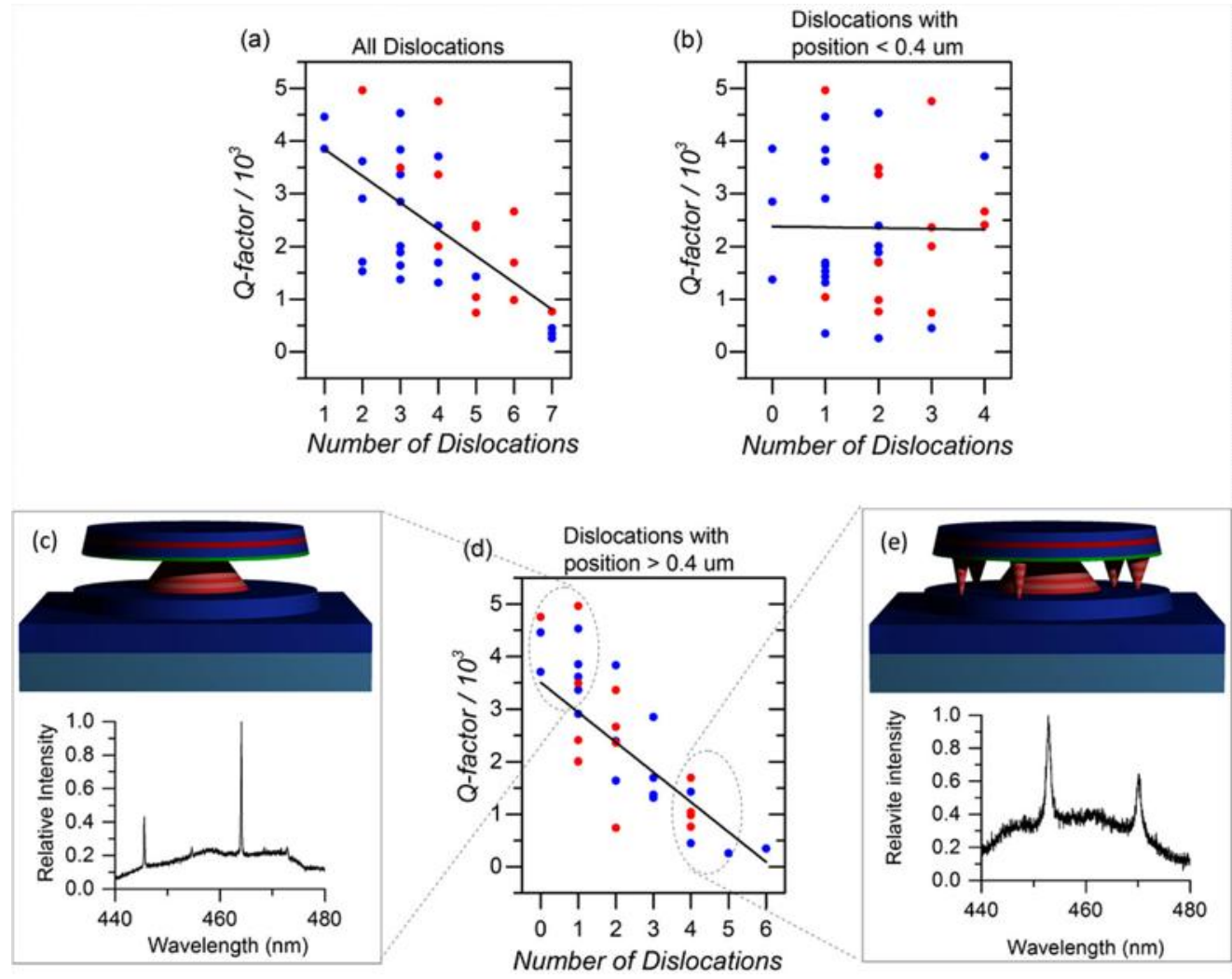

Figure 14: Microdisk Q-factor vs. threading dislocation number for radial positions: a) $0-0.6$ b) $<0.4$ d) $>0.4$ for $Q D$ (blue) and $Q W($ red) containing microdisks. c) and e) show a schematic of, and representative PL spectrum taken from microdisks of low and high dislocation number in the periphery of the disks [10].

The mechanisms by which the presence of TDs could influence microdisk Q-factor were investigated by Puchtler et al. through the use of finite difference time domain (FDTD) simulations. The presence of a whisker on the underside of a microdisk was modelled as a pyramidal protrusion with a range of dimensions and positions on the underside, producing results which suggest the Q-factor of the first order WGM decreases as the position of the whisker approaches the periphery of the microdisk and provides a radiative pathway for light confined in this region to escape [10].

Another important factor to consider is the effect of the dislocations themselves on the optical properties of the microdisks. TDs have been shown to increase impurity levels both at dislocation cores and in an extended region affected by the associated strain field during growth. TEM studies of dislocations have revealed strain related changes in doping occuring on the scale of several nanometres $[78,79]$. Threading dislocations can also contain vacancy states at dislocation cores giving rise to 'yellow-band' optical transitions, though these are far away from the wavelength range of III- 
nitride cavities [80,81]. In considering the impact of these factors on absorption in the micodisk, Puchtler et al. reported no significant effect on cavity Q-factor for linear attenuation coefficients up to $10^{9} \mathrm{~cm}^{-1}$ (several orders of magnitude greater than the expected value for highly doped GaN [82]) associated with dislocations modelled as a cylinder of radius $4 \mathrm{~nm}$. Hence, the effect of impurities or point defects associated with TDs on microdisk Q-factor is expected to be of only minor importance, due to the small volume of the cavity affected. Whilst non-radiative recombination at dislocation cores does affect quantum efficiency and is likely to have implications for electrically injected lasers, the lack of any substantial absorption of photons at dislocation sites means that dislocations per se have a rather limited effect on the Q-factor obtained by PL. It is thus the whiskers arising due to PEC etching which limit the Q-factor, rather than any inherent property of the TDs themselves, and from this perspective the use of alternative fabrication methods which avoid PEC rather than the elimination of TDs should be a priority for optimising device performance. Mexis et al. fabricated microdisks incorporating GaN QDs grown on AlN with AlGaN barriers with Q-factors reported as high as 7300 by growing the nitride structure on a silicon substrate, which can be selectively wet etched and produces whisker free microdisks despite the high dislocation density expected from growth on silicon [33]. In addition to the 'whisker-less' fabrication of high Q-factor micordisks, the growth of III-nitride optoelectronic structures on silicon substrates is particularly interesting with regards to the monolithic integration of nitride optoelectronics with silicon electronics, however there remain many difficulties in the large-area growth of $\mathrm{GaN}$ on $\mathrm{Si}$ due to the large lattice and thermal mismatch between epilayer and substrate [83].

\subsection{Effect of Dislocations on Microdisk Active Layers}

Although we have reviewed the effects of dislocations on the PEC etching process and the effect of unetched whiskers in microdisks on Q-factor, it is also important to consider the effect dislocations and defects may have on the active region in microdisk emitters which are typically QW or QD layers [84]. QD layers exhibit impacts of dislocations which are not relevant to QWs, and we will discuss these additional challenges here. 
Although Puchtler et al. reported little difference in Q-factors between microdisks fabricated with QW and QD layers [10], QD layers have attracted a large amount of interest due to the strong quantum confinement within these structures, allowing for large radiative recombination rates and extremely narrow linewidths. The theoretical advantages of QD layers compared to QW layers include lower-threshold, higher optical gain and superior temperature stability for lasing [50]. Single QD emitters have also been shown to be extremely promising for single photon source applications $[18,85]$.

The devices discussed in this review are have low densities of QDs, based on the estimated QD densities $\left(\sim 10^{9} \mathrm{~cm}^{-2}\right)$ from atomic force microscopy of the active layers prior to the GaN cap growth $[10,76]$ and the diameter of the microdisks $(\sim 1-2 \mu \mathrm{m})$. Low QD densities offer the opportunity for the observation of the coupling of single QWs to cavities, but in this context, the spectral and linewidth stability of the emission from the QD is crucial. Although experiments concerning QD-cavity coupling are often performed at cryogenic temperatures [86,87], it is worthwhile to note that temperatures above $40 \mathrm{~K}$ have been shown to significantly broaden the linewidth and shift the energy of some InGaN QDs [88]. Given that the effect of temperature can be roughly accounted for in cavity design and is not defect related, it will not be discussed in further detail here.

On the other hand, spectral diffusion on the order of seconds has been observed for InGaN QDs and shown to be both temperature independent up to $40 \mathrm{~K}$ and related to dislocations [89]. The Q-factors reported for microdisks with embedded InGaN QDs by Puchtler et al. indicate an optical mode with a FWHM spanning < $0.1 \mathrm{~nm}[10]$, comparable to the reported variation in the wavelength of emission (or spectral diffusion) from InGaN QDs on a timescale of several seconds [89] indicating such spectral diffusion may be a significant barrier to the observation of Purcell-enhanced emission and strong coupling in QD-containing microdisk cavities.

Reid et al. confirmed the role of dislocations in the spectral diffusion of InGaN QDs by comparing the magnitude of spectral diffusion across samples grown on templates with varying dislocation density [90]. Three GaN pseudo-substrates were grown for InGaN QD growth with low dislocation density (LDD), medium dislocation density (MDD) and high dislocation density (HDD). The temporal 
variation in QD emission was evaluated by acquiring consecutive spectra over time periods of an hour using two photon excitation in a time integrated $\mu$ PL (TIPL) set- up. Using the standard deviation of QD energy obtained via TIPL for 10 QDs in each sample Reid and co-authors reported a larger magnitude of spectral deviation for the HDD sample, as shown in Fig. 15.

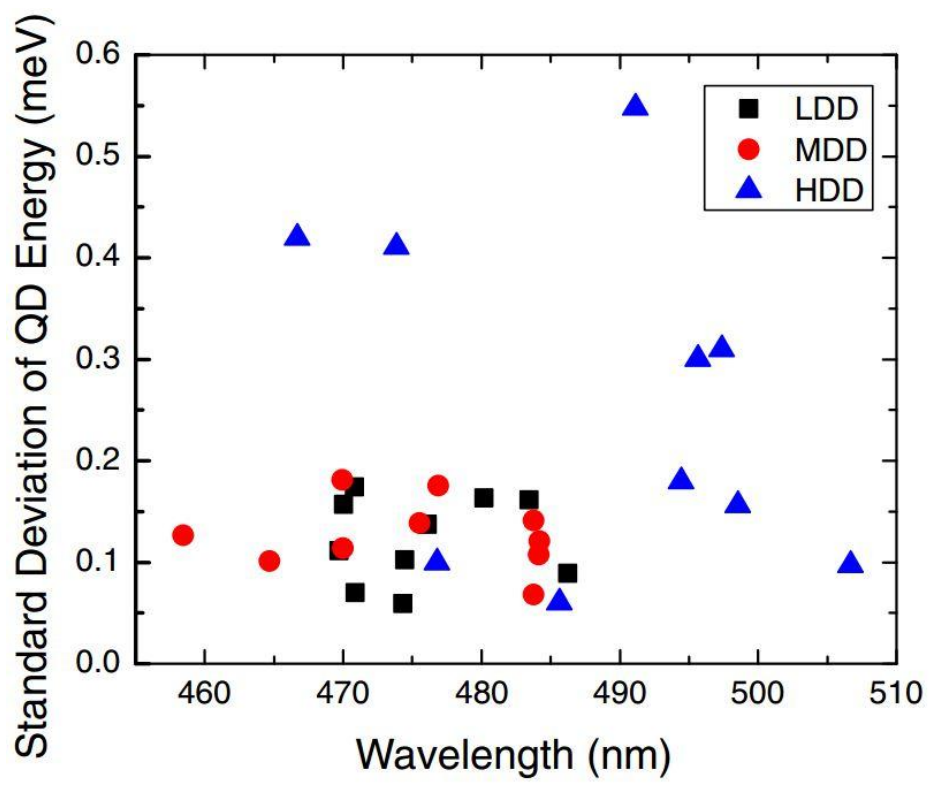

Figure 15: TIPL collected over 1 hour for 10 QDs for each sample. Standard deviation of QD emission energy against wavelength for 30 QDs. HDD QDs show a higher standard deviation on average. Copyright (2013) The Japan Society of Applied Physics [90].

The spectral diffusion in this case occurs over periods exceeding 5 seconds, which is the accumulation time for each TIPL acquisition. Although fluctuations of composition and thickness in the InGaN layer around the QDs can result in carrier localisation [91], only the trapping energy of dislocations should lead to a trapping lifetime on the order of seconds [92], supporting the correlation between dislocation density and QD spectral diffusion shown by Reid et al. [39]. As such the spectral diffusion in QD emission observed is attributed to trapped charges at dislocation, highlighting the potentially deleterious impact of dislocations on the microdisks devices based on QDs despite the limited impact of dislocation-induced absorption reported by Puchtler et al [10]. 


\section{Concluding Remarks}

Despite the effect of dislocations on microdisk Q-factor reported by Puchtler et al. and El-Ella et al., it is important to consider other factors which may affect microdisk Q-factors. Whilst an anticorrelation between microcavity Q-factor and dislocations counts within the modal volume of microdisk WGMs was observed by Puchtler et al., even dislocation-free microdisk Q-factors were observed to fall well short of theoretically predicted values, indicating whisker induced losses are not the primary limiting variable for microdisk Q-factor [10]. We will discuss the different contributions and primary limiting factor for cavity Q-value here.

The quality factor of a cavity can be described by the following equation:

$$
Q^{-1}=Q_{I A}^{-1}+Q_{r}^{-1}+Q_{s c a t}^{-1}
$$

where $\mathrm{Q}_{\mathrm{IA}}, \mathrm{Q}_{\mathrm{r}}$ and $\mathrm{Q}_{\text {scat }}$ denote the internal absorption losses, intrinsic radiative losses and surface scattering losses respectively. In discussing the relative contributions of each of these sources of loss in the III-nitride system it is important to note Q-factor is in part determined by cavity design and geometry. Intrinsic radiative losses decrease with increasing disk radius [93], indicating microdisks with smaller dimensions are in theory undesirable from this perspective. However low-threshold lasers require small modal volumes in order to increase the spontaneous emission factor, smaller microdisks with greater inherent radiative losses are required for these applications. Nonetheless, $\mathrm{Q}_{\mathrm{r}}$ is expected to be several orders of magnitude larger than the other factors for the microdisks described by Puchtler et al. and as such is not expected to affect the overall quality factor [10]. Surface scattering, which is dependent both on surface roughness resulting from the microdisk fabrication and disk morphology is also expected to play a minimal role in III-nitride microdisks, as the ICP etch process is capable of producing surfaces with an root-mean square (RMS) roughness below $2 \mathrm{~nm}$ [10]. It is in fact the losses due to internal absorption that are expected to dominate the Q-factor of IIInitride microdisks as emission below the band-edge of $\mathrm{GaN}$ can experience significant absorption due to moderate unintentional doping resulting in increased electronic transitions between donor-acceptor levels and defects [82]. Approximate $\mathrm{Q}_{\text {abs }}$ values calculated by Puchtler et al. for the wavelength of 
WGM observed in microdisks were in the range of $10^{3}-10^{4}$, depending on dopant levels, growth conditions and substrate $[82,94-96]$. These correspond roughly to the values reported, confirming the role of internal absorption as the determining factor for microdisk Q-value [10]. The large range of predicted Q-values is attributed to variations in structural defects and dopants in reported literature which can cause a wide variety of absorption in the free carrier absorption and Urbach tail regions [82]. Thus while microdisks in other materials systems such as silicon or GaAs have Q-values that are limited by intrinsic radiative and scattering losses and thus much higher (in the range of $10^{5}-10^{6}$ ) [93,97], III-nitride microdisk Q-factors are limited by absorption losses. This poses an extremely difficult challenge in terms of enhancing nitride microdisk Q-factor reducing internal absorption losses requires careful tailoring of both active region emission and background doping levels.

In conclusion, we have reviewed the effects of dislocations on the properties of III-nitride microdisk cavities. Interestingly, though the two main effects reviewed here have different implications for microdisk optimisation both are induced by the charge-trapping nature of dislocations. The reduction in surface hole concentration during the PEC etching process by dislocations has been shown to result in unetched whiskers on the underside of microdisks, which reduce microdisk Q-values if located at the outer-edge of the microdisk, where the first order WGMs reside. This same charge-trapping property which impedes PEC etching has been shown to induce instability in the emission of QDs, or spectral diffusion, a considerable obstacle to the achievement of both weak and strong coupling in QD embedded III-nitride microcavities. In addition to the effects of dislocations, internal absorption due to incorporated impurities also presents a considerable technological hurdle as the limiting variable on III-nitride microdisk Q-factor, suggesting that further optimisation strategies of III-nitride microdisks must combine careful engineering of emission wavelength and background doping levels, a fabrication approach which provides whisker-free microdisks and low dislocation densities for limited spectral diffusion in the context of integrated QDs in order to be truly effective.

\section{Acknowledgements}

The original research shown in this article has been funded by the European Research Council under the European Community's Seventh Framework Programme (FP7/2007-2013)/ ERC grant agreement 
no. 279361 (MACONS). RAO acknowledges the Royal Academy of Engineering Leverhulme Trust

Senior Research Fellowship scheme.

\section{References}

[1] Anon 2014 The Nobel Prize in Physics 2014 Nobel Media

[2] Dorsaz J, Carlin J F, Zellweger C M, Gradecak S and Ilegems M 2004 InGaN/GaN resonantcavity LED including an AlInN/GaN Bragg mirror Phys. Status Solidi Appl. Res. 201 2675-8

[3] Lu T-C, Kao C-C, Kuo H-C, Huang G-S and Wang S-C $2008 \mathrm{CW}$ lasing of current injection blue GaN-based vertical cavity surface emitting laser Appl. Phys. Lett. 92141102

[4] Oliver R A, Jarjour A F, Taylor R A, Tahraoui A, Zhang Y, Kappers M J and Humphreys C J 2008 Growth and assessment of InGaN quantum dots in a microcavity: A blue single photon source Mater. Sci. Eng. B 147 108-13

[5] Christopoulos S, von Högersthal G B H, Grundy a. J D, Lagoudakis P G, Kavokin a. V., Baumberg J J, Christmann G, Butté R, Feltin E, Carlin J-F and Grandjean N 2007 RoomTemperature Polariton Lasing in Semiconductor Microcavities Phys. Rev. Lett. 98126405

[6] Bhattacharya P, Frost T, Deshpande S, Baten M Z, Hazari A and Das A 2014 Room temperature electrically injected polariton laser Phys. Rev. Lett. 112 29-31

[7] Tamboli A C, Haberer E D, Sharma R, Lee K H, Nakamura S and Hu E L 2007 Roomtemperature continuous-wave lasing in GaN/InGaN microdisks Nat. Photonics 1 61-4

[8] Triviño N V, Butté R, Carlin J-F and Grandjean N 2015 Continuous Wave Blue Lasing in IIINitride Nanobeam Cavity on Silicon Nano Lett. 15 1259-63

[9] Niu N, Woolf A, Wang D, Zhu T, Quan Q, Oliver R a. and Hu E L 2015 Ultra-low threshold gallium nitride photonic crystal nanobeam laser Appl. Phys. Lett. 106231104

[10] Puchtler T J, Woolf A, Zhu T, Gachet D, Hu E L and Oliver R a. 2015 Effect of Threading Dislocations on the Quality Factor of InGaN/GaN Microdisk Cavities ACS Photonics 2 13743

[11] Bennett S E 2010 Dislocations and their reduction in GaN Mater. Sci. Technol. 26 1017-28

[12] Zhang Y, Zhang X, Li K H, Cheung Y F, Feng C and Choi H W 2015 Advances in III-nitride semiconductor microdisk lasers Phys. Status Solidi 212 960-73

[13] Aharonovich I, Niu N, Rol F, Russell K J, Woolf A, El-Ella H a. R, Kappers M J, Oliver R a and $\mathrm{Hu}$ E L 2011 Controlled tuning of whispering gallery modes of GaN/InGaN microdisk cavities Appl. Phys. Lett. 99111111

[14] Aharonovich I, Woolf A, Russell K J, Zhu T, Niu N, Kappers M J, Oliver R a. and Hu E L 2013 Low threshold, room-temperature microdisk lasers in the blue spectral range Appl. Phys. Lett. 103021112

[15] Niu N, Liu T-L, Aharonovich I, Russell K J, Woolf A, Sadler T C, El-Ella H a. R, Kappers M J, Oliver R a. and Hu E L 2012 A full free spectral range tuning of p-i-n doped gallium nitride microdisk cavity Appl. Phys. Lett. 101161105

[16] Gayral B, Gérard J M, Sermage B, Lemaitre a. and Dupuis C 2001 Time-resolved probing of 
the Purcell effect for InAs quantum boxes in GaAs microdisks Appl. Phys. Lett. 78 2828-30

[17] Vahala K J 2003 Optical microcavities. Nature 424 839-46

[18] Jarjour A F, Taylor R a., Oliver R a., Kappers M J, Humphreys C J and Tahraoui A 2007 Cavity-enhanced blue single-photon emission from a single InGaN $/ G a N$ quantum dot Appl. Phys. Lett. 91052101

[19] Malpuech G, Di Carlo A, Kavokin A, Baumberg J J, Zamfirescu M and Lugli P 2002 Roomtemperature polariton lasers based on GaN microcavities Appl. Phys. Lett. $81412-4$

[20] Imamoglu a, Awschalom D, Burkard G, DiVincenzo D P, Loss D, Sherwin M and Small a 1999 Quantum information processing using quantum dot spins and cavity QED Phys. Rev. Lett. 83 4204-7

[21] Hennessy K, Badolato a., Winger M, Gerace D, Atatüre M, Gulde S, Fält S, Hu E L and Imamoğlu a. 2007 Quantum nature of a strongly coupled single quantum dot-cavity system Nature 445 896-9

[22] Aleksiejūnas R, Sūdžius M, Malinauskas T, Vaitkus J, Jarašiūnas K and Sakai S 2003 Determination of free carrier bipolar diffusion coefficient and surface recombination velocity of undoped GaN epilayers Appl. Phys. Lett. 831157

[23] Muth J F, Lee J H, Shmagin I K, Kolbas R M, Casey H C, Keller B P, Mishra U K and DenBaars S P 1997 Absorption coefficient, energy gap, exciton binding energy, and recombination lifetime of $\mathrm{GaN}$ obtained from transmission measurements Appl. Phys. Lett. 71 2572

[24] Ramvall P, Tanaka S, Nomura S, Riblet P and Aoyagi Y 1998 Observation of confinementdependent exciton binding energy of GaN quantum dots Appl. Phys. Lett. 731104

[25] Khan A, Balakrishnan K and Katona T 2008 Ultraviolet light-emitting diodes based on group three nitrides Nat. Photonics 2 77-84

[26] Nakamura S, Mukai T and Senoh M 1991 High-Power GaN P-N Junction Blue-LightEmitting Diodes Jpn. J. Appl. Phys. 30 1998-2001

[27] Someya T, Werner R, Forchel A, Catalano M, Cingolani R and Arakawa Y 1999 Room Temperature Lasing at Blue Wavelengths in Gallium Nitride Microcavities Science (80-. ). 285 $1905-6$

[28] Reid B P L, Kocher C, Zhu T, Oehler F, Emery R, Chan C C S, Oliver R a. and Taylor R a. 2014 Observations of Rabi oscillations in a non-polar InGaN quantum dot Appl. Phys. Lett. 104263108

[29] Bhattacharya P, Xiao B, Das A, Bhowmick S and Heo J 2013 Solid State Electrically Injected Exciton-Polariton Laser Phys. Rev. Lett. 110206403

[30] Schneider C, Rahimi-Iman A, Kim N Y, Fischer J, Savenko I G, Amthor M, Lermer M, Wolf A, Worschech L, Kulakovskii V D, Shelykh I a., Kamp M, Reitzenstein S, Forchel A, Yamamoto Y and Höfling S 2013 An electrically pumped polariton laser Nature 497 348-52

[31] Richard M, Kasprzak J, Romestain R, André R and Dang L S 2005 Spontaneous Coherent Phase Transition of Polaritons in CdTe Microcavities Phys. Rev. Lett. 94187401

[32] Saba M, Ciuti C, Bloch J, Thierry-Mieg V, André R, Dang L S, Kundermann S, Mura a., Bongiovanni G, Staehli J L and Deveaud B 2001 High-temperature ultrafast polariton parametric amplification in semiconductor microcavities. Nature $\mathbf{4 1 4} 731-5$

[33] Mexis M, Sergent S, Guillet T, Brimont C, Bretagnon T, Gil B, Semond F, Leroux M, Néel D, David S, Chécoury X and Boucaud P 2011 High quality factor nitride-based optical cavities: microdisks with embedded $\mathrm{GaN} / \mathrm{Al}(\mathrm{Ga}) \mathrm{N}$ quantum dots. Opt. Lett. 36 2203-5 
[34] Guillet T, Mexis M, Sergent S, Néel D, Rennesson S, Brimont C, Bretagnon T, Gil B, SamGiao D, Gayral B, Semond F, Leroux M, David S, Checoury X and Boucaud P 2012 High quality factor photonic resonators for nitride quantum dots Phys. Status Solidi 249 449-54

[35] Sergent S, Arita M, Kako S, Iwamoto S and Arakawa Y 2012 High-Q (\&gt;5000) AlN nanobeam photonic crystal cavity embedding GaN quantum dots Appl. Phys. Lett. 100121103

[36] Kao C-C, Huang H ., Tsai J ., Yu C ., Lin C ., Kuo H . and Wang S . 2004 Study of dry etching for $\mathrm{GaN}$ and $\mathrm{InGaN}$-based laser structure using inductively coupled plasma reactive ion etching Mater. Sci. Eng. B 107 283-8

[37] Ren C X 2015 Polarisation fields in III-nitrides: effects and control Mater. Sci. Technol. 00 1743284715 Y.000

[38] Rice J H, Robinson J W, Jarjour A, Taylor R a., Oliver R a., Andrew G, Briggs D, Kappers M J and Humphreys C J 2004 Temporal variation in photoluminescence from single InGaN quantum dots Appl. Phys. Lett. 84 4110-2

[39] Ã B P L R, Zhu T, Puchtler T J, Fletcher L J, Chan C C S, Oliver R A and Taylor R A Origins of Spectral Diffusion in the Micro-Photoluminescence of Single InGaN Quantum Dots Origins of Spectral Diffusion in the Micro-Photoluminescence of Single InGaN Quantum Dots 01

[40] Scholz F 2012 Semipolar GaN grown on foreign substrates: a review Semicond. Sci. Technol. 27024002

[41] Nakamura S 1998 The Roles of Structural Imperfections in InGaN-Based Blue Light-Emitting Diodes and Laser Diodes Science (80-. ). 281 956-61

[42] Watson-Parris D, Godfrey M J, Dawson P, Oliver R a., Galtrey M J, Kappers M J and Humphreys C J 2011 Carrier localization mechanisms in $\mathrm{In}_{-}\{\mathrm{x}\} \mathrm{Ga} \_\{1-\mathrm{x}\} \mathrm{N} / \mathrm{GaN}$ quantum wells Phys. Rev. B $\mathbf{8 3} 115321$

[43] Hangleiter a., Hitzel F, Netzel C, Fuhrmann D, Rossow U, Ade G and Hinze P 2005 Suppression of nonradiative recombination by V-shaped pits in GaInN/GaN quantum wells produces a large increase in the light emission efficiency Phys. Rev. Lett. 95 1-4

[44] Li Y, Yun F, Su X, Liu S, Ding W and Hou X 2014 Deep hole injection assisted by large Vshape pits in InGaN/GaN multiple-quantum-wells blue light-emitting diodes J. Appl. Phys. 116 123101

[45] Tomiya S, Hino T, Goto S, Takeya M and Ikeda M 2004 Dislocation Related Issues in the Degradation of GaN-Based Laser Diodes IEEE J. Sel. Top. Quantum Electron. 10 1277-86

[46] Chen R, Sun H D, Wang T, Hui K N and Choi H W 2010 Optically pumped ultraviolet lasing from nitride nanopillars at room temperature Appl. Phys. Lett. 96241101

[47] Carlin J-F, Zellweger C, Dorsaz J, Nicolay S, Christmann G, Feltin E, Butté R and Grandjean N 2005 Progresses in III-nitride distributed Bragg reflectors and microcavities using AlInN/GaN materials Phys. Status Solidi 242 2326-44

[48] Lin C-H, Wang J-Y, Chen C-Y, Shen K-C, Yeh D-M, Kiang Y-W and Yang C C 2011 A GaN photonic crystal membrane laser Nanotechnology 22025201

[49] Arita M, Ishida S, Kako S, Iwamoto S and Arakawa Y 2007 AlN air-bridge photonic crystal nanocavities demonstrating high quality factor Appl. Phys. Lett. 91051106

[50] Woolf a., Puchtler T, Aharonovich I, Zhu T, Niu N, Wang D, Oliver R and Hu E L 2014 Distinctive signature of indium gallium nitride quantum dot lasing in microdisk cavities Proc. Natl. Acad. Sci. 111 14042-6

[51] McCall S L, Levi a. F J, Slusher R E, Pearton S J and Logan R a. 1992 Whispering-gallery mode microdisk lasers Appl. Phys. Lett. 60289 
[52] Gayral B, Gérard J M, Lemaître a., Dupuis C, Manin L and Pelouard J L 1999 High-Q wetetched GaAs microdisks containing InAs quantum boxes Appl. Phys. Lett. 751908

[53] Simeonov D, Feltin E, Bühlmann H-J, Zhu T, Castiglia a., Mosca M, Carlin J-F, Butté R and Grandjean N 2007 Blue lasing at room temperature in high quality factor GaNAlInN microdisks with InGaN quantum wells Appl. Phys. Lett. 90061106

[54] Haberer E D, Sharma R, Stonas a. R, Nakamura S, DenBaars S P and Hu E L 2004 Removal of thick $(\& \mathrm{gt} ; 100 \mathrm{~nm})$ InGaN layers for optical devices using band-gap-selective photoelectrochemical etching Appl. Phys. Lett. 85762

[55] Choi H W, Hui K N, Lai P T, Chen P, Zhang X H, Tripathy S, Teng J H and Chua S J 2006 Lasing in GaN microdisks pivoted on Si Appl. Phys. Lett. 89 2006-8

[56] Kouno T, Kishino K and Sakai M 2011 Lasing Action on Whispering Gallery Mode of SelfOrganized GaN Hexagonal Microdisk Crystal Fabricated by RF-Plasma-Assisted Molecular Beam Epitaxy IEEE J. Quantum Electron. 47 1565-70

[57] Baek H, Lee C-H, Chung K and Yi G-C 2013 Epitaxial GaN Microdisk Lasers Grown on Graphene Microdots. Nano Lett. 13 2782-5

[58] Simeonov D, Feltin E, Altoukhov a., Castiglia a., Carlin J F, Butt́ R and Grandjean N 2008 High quality nitride based microdisks obtained via selective wet etching of AlInN sacrificial layers Appl. Phys. Lett. 92 2008-10

[59] Haberer E D, Sharma R, Meier C, Stonas a. R, Nakamura S, DenBaars S P and Hu E L 2004 Free-standing, optically pumped, GaNhnaN microdisk lasers fabricated by photoelectrochemical etching Appl. Phys. Lett. 855179

[60] Youtsey C, Romano L T and Adesida I 1998 Gallium nitride whiskers formed by selective photoenhanced wet etching of dislocations Appl. Phys. Lett. 73797

[61] Chang S, Rex N B, Chang R K, Chong G and Guido L J 1999 Stimulated emission and lasing in whispering-gallery modes of GaN microdisk cavities Appl. Phys. Lett. 75166

[62] Minsky M S, White M and Hu E L 1996 Room-temperature photoenhanced wet etching of GaN Appl. Phys. Lett. 68 1531-3

[63] Visconti P, Reshchikov M a., Jones K M, Wang D F, Cingolani R, Morkoç̌ H, Molnar R J and Smith D J 2001 Highly selective photoelectrochemical etching of nitride materials for defect investigation and device fabrication J. Vac. Sci. Technol. B Microelectron. Nanom. Struct. 19 1328

[64] Youtsey C, Romano L T, Molnar R J and Adesida I 1999 Rapid evaluation of dislocation densities in n-type GaN films using photoenhanced wet etching Appl. Phys. Lett. 743537

[65] Youtsey C, Adesida I and Bulman G 1997 Highly anisotropic photoenhanced wet etching of n-type GaN Appl. Phys. Lett. 712151

[66] Stonas a. R, Margalith T, DenBaars S P, Coldren L a. and Hu E L 2001 Development of selective lateral photoelectrochemical etching of $\mathrm{InGaN} / \mathrm{GaN}$ for lift-off applications Appl. Phys. Lett. 781945

[67] Lazar S, Weyher J L, Macht L, Tichelaar F D and Zandbergen H W 2004 Nanopipes in GaN : photo-etching and TEM study Eur. Phys. J. Appl. Phys. 27 275-8

[68] Rosner S J, Carr E C, Ludowise M J, Girolami G and Erikson H I 1997 Correlation of cathodoluminescence inhomogeneity with microstructural defects in epitaxial GaN grown by metalorganic chemical-vapor deposition Appl. Phys. Lett. 70420

[69] Sugahara T, Sato H, Hao M, Naoi Y, Kurai S, Tottori S, Yamashita K, Nishino K, Romano L T and Sakai S 1998 Direct Evidence that Dislocations are Non-Radiative Recombination 
Centers in GaN Jpn. J. Appl. Phys. 37 L398-400

[70] Cherns D and Jiao C 2001 Electron Holography Studies of the Charge on Dislocations in GaN Phys. Rev. Lett. 87205504

[71] Bals S, Tirry W, Geurts R, Yang Z and Schryvers D 2007 High-quality sample preparation by low kV FIB thinning for analytical TEM measurements. Microsc. Microanal. 13 80-6

[72] Griffiths J T, Zhang S, Rouet-Leduc B, Fu W Y, Bao A, Zhu D, Wallis D J, Howkins A, Boyd I, Stowe D, Kappers M J, Humphreys C J and Oliver R A 2015 Nanocathodoluminescence Reveals Mitigation of the Stark Shift in InGaN Quantum Wells by Si Doping Nano Lett. 151022122922003

[73] Midgley P a and Dunin-Borkowski R E 2009 Electron tomography and holography in materials science. Nat. Mater. 8 271-80

[74] Vico Triviño N, Rossbach G, Dharanipathy U, Levrat J, Castiglia a., Carlin J-F, Atlasov K a., Butté R, Houdré R and Grandjean N 2012 High quality factor two dimensional GaN photonic crystal cavity membranes grown on silicon substrate Appl. Phys. Lett. 100071103

[75] El-Ella H a. R, Rol F, Collins D P, Kappers M J, Taylor R a., Hu E L and Oliver R a. 2011 InGaN super-lattice growth for fabrication of quantum dot containing microdisks $J$. Cryst. Growth 321 113-9

[76] El-Ella H a. R, Rol F, Kappers M J, Russell K J, Hu E L and Oliver R a. 2011 Dislocation density-dependent quality factors in InGaN quantum dot containing microdisks Appl. Phys. Lett. 98131909

[77] Srinivasan S, Geng L, Liu R, Ponce F a., Narukawa Y and Tanaka S 2003 Slip systems and misfit dislocations in InGaN epilayers Appl. Phys. Lett. 83 5187-9

[78] Rhode S K, Horton M K, Kappers M J, Zhang S, Humphreys C J, Dusane R O, Sahonta S L and Moram M a. $2013 \mathrm{Mg}$ doping affects dislocation core structures in GaN Phys. Rev. Lett. $1111-4$

[79] Horton M K, Rhode S, Sahonta S-L, Kappers M J, Haigh S J, Pennycook T J, Humphreys C J, Dusane R O and Moram M A 2015 Segregation of In to dislocations in InGaN. Nano Lett. 15 923-30

[80] Xin Y, James E M, Arslan I, Sivananthan S, Browning N D, Pennycook S J, Omnès F, Beaumont B, Faurie J-P and Gibart P 2000 Direct experimental observation of the local electronic structure at threading dislocations in metalorganic vapor phase epitaxy grown wurtzite GaN thin films Appl. Phys. Lett. 76466

[81] Elsner J, Jones R, Sitch P K, Porezag V D, Elstner M, Frauenheim T, Heggie M I, Oberg S and Briddon P R 1997 Theory of Threading Edge and Screw Dislocations in GaN Phys. Rev. Lett. 79 3672-5

[82] Ambacher O, Rieger W, Ansmann P, Angerer H, Moustakas T D and Stutzmann M 1996 Subbandgap absorption of gallium nitride determined by photothermal deflection spectroscopy Solid State Commun. 97 365-70

[83] Zhu D, Wallis D J and Humphreys C J 2013 Prospects of III-nitride optoelectronics grown on Si. Rep. Prog. Phys. 76106501

[84] Zhang Y, Zhang X, Li K H, Cheung Y F, Feng C and Choi H W 2015 Advances in III-nitride semiconductor microdisk lasers. Phys. Status Solidi A Appl. Mater. Sci. 212 960-73

[85] Kako S, Santori C, Hoshino K, Götzinger S, Yamamoto Y and Arakawa Y 2006 A gallium nitride single-photon source operating at 200 K. Nat. Mater. 5 887-92

[86] Vučković J, Fattal D, Santori C, Solomon G S and Yamamoto Y 2003 Enhanced single- 
photon emission from a quantum dot in a micropost microcavity Appl. Phys. Lett. 82 3596-8

[87] Reithmaier J P, Sek G, Löffler a, Hofmann C, Kuhn S, Reitzenstein S, Keldysh L V, Kulakovskii V D, Reinecke T L and Forchel a 2004 Strong coupling in a single quantum dotsemiconductor microcavity system. Nature 432 197-200

[88] Moriwaki O, Someya T, Tachibana K, Ishida S and Arakawa Y 2000 Narrow photoluminescence peaks from localized states in InGaN quantum dot structures Appl. Phys. Lett. 762361

[89] Rice J H, Robinson J W, Jarjour A, Taylor R a., Oliver R a., Briggs G A D, Kappers M J and Humphreys C J 2004 Temporal variation in photoluminescence from single InGaN quantum dots Appl. Phys. Lett. 844110

[90] Reid B P L, Zhu T, Puchtler T J, Fletcher L J, Chan C C S, Oliver R A and Taylor R A 2013 Origins of Spectral Diffusion in the Micro-Photoluminescence of Single InGaN Quantum Dots Jpn. J. Appl. Phys. 52 08JE01

[91] Oliver R A, Massabuau F C-P, Kappers M J, Phillips W a., Thrush E J, Tartan C C, Blenkhorn W E, Badcock T J, Dawson P, Hopkins M a., Allsopp D W E and Humphreys C J 2013 The impact of gross well width fluctuations on the efficiency of GaN-based light emitting diodes Appl. Phys. Lett. 103141114

[92] Seufert J, Weigand R, Bacher G, Kümmell T, Forchel a., Leonardi K and Hommel D 2000 Spectral diffusion of the exciton transition in a single self-organized quantum dot Appl. Phys. Lett. 761872

[93] Borselli M, Johnson T and Painter O 2005 Beyond the Rayleigh scattering limit in high-Q silicon microdisks: theory and experiment. Opt. Express 13 1515-30

[94] Omnès F, Marenco N, Beaumont B, de Mierry P, Monroy E, Calle F and Muñoz E 1999 Metalorganic vapor-phase epitaxy-grown AlGaN materials for visible-blind ultraviolet photodetector applications J. Appl. Phys. 865286

[95] Yu G, Wang G, Ishikawa H, Umeno M, Soga T, Egawa T, Watanabe J and Jimbo T 1997 Optical properties of wurtzite structure $\mathrm{GaN}$ on sapphire around fundamental absorption edge $(0.78-4.77 \mathrm{eV})$ by spectroscopic ellipsometry and the optical transmission method Appl. Phys. Lett. 703209

[96] Strite, S., Morkoç H 1992 GaN, AlN, and InN: A review J. Vac. Sci. Technol. B 10 1237-66

[97] Srinivasan K and Painter O 2007 Linear and nonlinear optical spectroscopy of a strongly coupled microdisk-quantum dot system Nature 450 862-5 